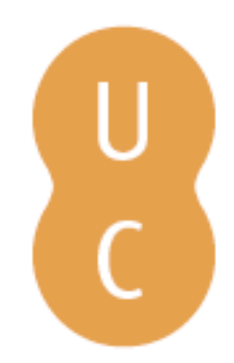

\title{
nombalina
}

\section{Las sonatas de Carlos Seixas dentro de la música de tecla de la Península lbérica en} el siglo XVIII

\author{
Autor(es): López-Calo, José \\ Publicado por: Imprensa da Universidade de Coimbra \\ URL \\ persistente: URI:http://hdl.handle.net/10316.2/32517 \\ DOI: $\quad$ DOI:http://dx.doi.org/10.14195/978-989-26-0409-1_5 \\ Accessed : $\quad$ 26-Apr-2023 10:02:38
}

A navegação consulta e descarregamento dos títulos inseridos nas Bibliotecas Digitais UC Digitalis, UC Pombalina e UC Impactum, pressupõem a aceitação plena e sem reservas dos Termos e Condições de Uso destas Bibliotecas Digitais, disponíveis em https://digitalis.uc.pt/pt-pt/termos.

Conforme exposto nos referidos Termos e Condições de Uso, o descarregamento de títulos de acesso restrito requer uma licença válida de autorização devendo o utilizador aceder ao(s) documento(s) a partir de um endereço de IP da instituição detentora da supramencionada licença.

Ao utilizador é apenas permitido o descarregamento para uso pessoal, pelo que o emprego do(s) título(s) descarregado(s) para outro fim, designadamente comercial, carece de autorização do respetivo autor ou editor da obra.

Na medida em que todas as obras da UC Digitalis se encontram protegidas pelo Código do Direito de Autor e Direitos Conexos e demais legislação aplicável, toda a cópia, parcial ou total, deste documento, nos casos em que é legalmente admitida, deverá conter ou fazer-se acompanhar por este aviso.

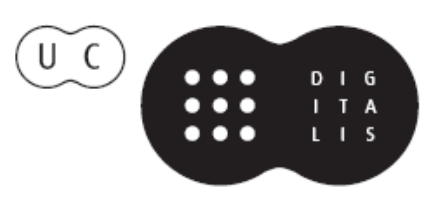




\section{J. M. PEDROSA CARDOSO}

Coordenação

J. M. PEDROSA CARDOSO • ANTÓNIO FILIPE PIMENTEL MANUEL CARLOS DE BRITO • JOSÉ LÓPEZ-CALO JOSÉ EDUARDO MARTINS • ABÍLIO QUEIRÓS

Autores

\section{Carlos Seixas, \\ de Coimbra}

Ano Seixas

Exposição Documental

Coimbra I mprensa da Universidade $\quad 2004$ 
(Página deixada propositadamente em branco) 
J. M. PEDROSA CARDOSO

Coordenação

J. M. PEDROSA CARDOSO • ANTÓNIO FLLIPE PIMENTEL

MANUEL CARLOS DE BRITO • JOSÉ LÓPEZ-CALO

JOSÉ EDUARDO MARTINS • ABÍLIO QUEIRÓS

Autores

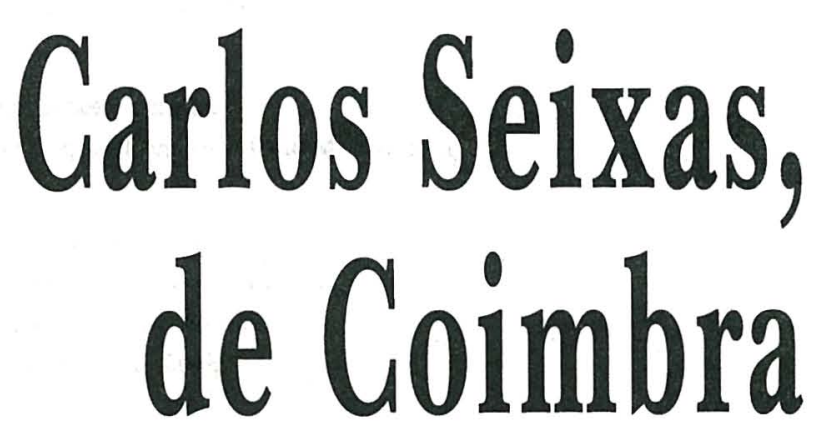

Ano Seixas

Exposição Documental

Coimbra - Imprensa da Universidade $\quad 2004$ 


\section{Coordenação editorial}

Imprensa da Universidade de Coimbra

\section{Concepção gráfica}

António Barros

\section{Paginação}

Victor Hugo Fernandes

\section{Créditos fotográficos}

p. 98 José Manuel Vasconcellos, p. 99 e 100 Varela Pécurto

\section{Execução gráfica}

Imprensa de Coimbra, Lda.

Couraça dos Apóstolos, 126

3000-372 Coimbra

\section{ISBN}

972-8704-33-X

\section{Depósito Legal}

\section{$218421 / 04$}

(C) 2004, Imprensa da Universidade de Coimbra

Obra publicada com o patocínio do GRUPO AMORIM:

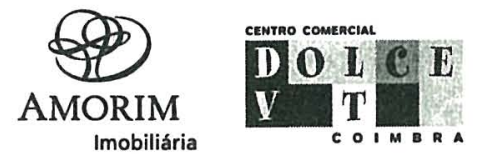

Apoio de:

Reitoria da Universidade de Coimbra

Biblioteca da Universidade de Coimbra

Arquivo da Universidade de Coimbra

Faculdade de Letras da Universidade de Coimbra 


\section{Las sonatas de Carlos Seixas \\ dentro de la música de tecla de la Península Ibérica \\ EN EL SIGLO XVIII}

\section{José López-Calo}

(Universidad de Santiago de Compostela)

Deseo comenzar recordando a don Santiago Kastner, maestro de maestros, a quien tanto debemos todos los que nos interesamos por la música ibérica de otros tiempos y que para mí ha sido siempre maestro venerado y luz e inspiración en mis estudios de musicología. Para el tema del presente simposio este recuerdo es particularmente debido, ya que Kastner fue quien, en verdad, descubrió a Seixas, publicó una biografía de Seixas, verdaderamente modélica ${ }^{(1)}$, publicó varias de sus sonatas y otras obras musicales, amén de descubrir la mayoría de las fuentes históricas donde se conservan las obras del gran maestro portugués del siglo XVIII. Por eso deseo ofrecer a su memoria esta pequeña contribución mía a los estudios en torno a ese gran organista y compositor.

Deseo igualmente agradecer a la Universidad de Coimbra y a los organizadores de este simposio, en particular al Excmo. Sr. Pro-Reitor de la Universidad, Prof. Dr. D. João Gouveia Monteiro, y al coordinador, Prof. Dr. D. José María Pedrosa Cardoso, el honor que me han conferido invitándome a participar en estas sesiones de estudio, y precisamente a contribuir a ellas con la ponencia inaugural.

(1) Santiago Kastner: Carlos de Scixas. Coimbra: Coimbra Editora, Limitada, 1947. 
Este agradecimiento tiene un importante motivo añadido: mi asistencia a este evento me permite retomar los contactos con los colegas portugueses y hasta, si puedo expresarme así, volver a mis raíces, cuando, hace ya casi un cuarto de siglo, iniciamos, precisamente en esta veneranda Universidad de Coimbra, aquel bellísimo proyecto de los "Encuentros de Musicología Ibérica”, en que los musicólogos de ambas naciones nos reuníamos alternativamente en España y en Portugal, para convivir aquellos días inolvidables y estudiar juntos algunos de los numerosos temas de nuestra común historia musical y cultural.

Más tarde los avatares de la vida nos han separado. Pero yo sigo añorando aquellos proyectos, aquellas conversaciones, aquellas ansias de futuro que todos teníamos entonces. En mi corazón y en mi recuerdo sigue perfectamente vivo todo aquel mundo de ilusiones y de deseos. Por eso considero esta invitación, y el hecho de que yo me encuentre hoy entre ustedes, no solamente un honor, un altísimo honor, sino también una gran alegría y hasta, si se me permite, un soñar en que quizá puedan volver días en que caminemos más juntos de lo que hemos estado que en los últimos años.

Finalmente, deseo pedir excusas por expresarme en español. No solamente leo con toda comodidad el portugués, sino que también lo escribo con cierta corrección. En cambio, debo confesar que la fonética portuguesa se me resiste no poco, por lo que he preferido redactar mi ponencia en español, que espero todos entiendan sin especial dificultad.

En torno al año 1700 la música religiosa ibérica, tanto en España como en Portugal, y, en buena medida, en Iberoamérica, experimentó el cambio más profundo e importante de su historia. Si se fuera a buscar un término que, al margen de las descripciones técnicas de los elementos que cambiaron, y del signo en que cambiaron, definiese gráficamente ese cambio, habría que decir que con el siglo XVII terminaba la música antigua y con el XVIII comenzaba la moderna.

Es ésta, por supuesto, una definición un poco simplista, pero en su expresión generalizante resume perfectamente en qué consistió el cambio: la música que hasta ese momento se interpretaba en nuestras catedrales que era casi la única que existía, al revés de lo que pasaba en otras naciones 
europeas, sobre todo en Italia, - era tan sólo el fruto, la consecuencia, de una evolución de siglos, generada por las fuerzas internas de la música, sin apenas influjo externo; y esto en todos los elementos constitutivos de la música, desde la melodía, sus intervalos y sus ritmos, hasta la concepción de las composiciones y las formas musicales. Cambiaron igualmente los instrumentos, pasando, también ellos, y de modo muy visible, de los "antiguos" - chirimías, bajoncillos, cornetas, sacabuches... - a los modernos, primeros de todos los violines, así como los oboes, las flautas - primero las rectas, herederas directas de las antiguas, aunque construidas, y sobre todo usadas, en un modo del todo nuevo, pero que pronto quedaron suplantadas por las traveseras -, los clarines, las trompas...

Todo ello cambió desde a partir de hacia 1700, iniciándose entonces una música del todo nueva. Durante un tiempo convivieron, en parte, ambos estilos, pero el nuevo se impuso con rapidez y acabó suplantando totalmente al anterior en el giro de muy pocos años.

Suele atribuirse este cambio a la entrada de la música italiana en España, debida, sobre todo, al influjo de las dos esposas de Felipe V: María Luisa de Saboya primero, y, tras enviudar el rey en 1714, Isabel de Farnesio, con la que contrajo matrimonio el mismo año. En Portugal el cambio tuvo lugar aproximadamente por las mismas fechas; y si en este punto los asuntos de la Corte de Madrid no ejercieron particular influjo en el país vecino, sí lo ejercieron otros hechos, sobre todo el cosmopolitismo de la corte de Lisboa, que entonces, como antes y después, era muy intenso, siendo más activas que en la de Madrid las relaciones con Italia y su cultura, también la musical. El hecho es que por entonces comienzan a hacerse habituales músicos italianos en determinadas capillas musicales de las catedrales portuguesas, lo mismo que sucedía en algunas españolas. Ya los había de antes, pero en mucho menor número. Naturalmente, la ópera italiana, que entró poco después de comenzado el siglo, tanto en España como en Portugal, acrecentó, de modo decisivo y ya irreversible, ese influjo de la música italiana sobre la ibérica.

Así, pues, que hubo ese influjo es evidente. Y con todo yo me pregunto si el concepto que habitualmente se tiene y que viene repetido en las historias y hasta en artículos monográficos, del influjo italiano en este cambio en la música de la península ibérica, no debiera ser matizado. Confieso que no he tenido ocasión de estudiar en profundidad lo sucedido en Portugal en estos años críticos de finales del siglo XVII y primer cuarto del XVIII, 
también porque fuera de la benemérita iniciativa de la Fundación Gulbenkian, con su monumental "Portugaliae Musica", apenas se ha publicado música religiosa portuguesa de esa época; por otra parte, el interés de los coordinadores de la gran colección lusa se centraba más bien en otra música, que era más urgente rescatar, por lo que las composiciones que hubieran interesado en este punto son poco menos que desconocidas, y sería necesario ir a los archivos para estudiarlas.

Quizá fuera ésta una buena ocasión para que los colegas portugueses se propusieran comenzar una recuperación sistemática de esta importante parcela de nuestra historia artística y cultural.

Afortunadamente, esa falta la suplen, en cierto modo, los catálogos publicados por el canónigo don José Augusto Alegría. Lástima grande que de las catedrales sólo haya publicado el de la de Évora. Ojalá que alguien pronto acometa la publicación de los de las demás catedrales, y quizá con un criterio organizativo un poco más coherente que el que él siguió en el suyo. De todas formas, por los catálogos que él publicó, el de Évora y el de las dos grandes bibliotecas públicas, se ve, con toda precisión, que el proceso evolutivo de la música religiosa en ambas naciones de la península es perfectamente coetáneo y siempre con idéntica orientación; de tal manera, que más que hablar de música española o portuguesa se debería hablar de música ibérica, que es igual en las dos naciones - y lo mismo se debe decir de Iberoamérica -, y que es diferente de la de cualquier otra nación europea. De todas formas, fuerza es confesar que se está muy lejos - y ciertamente yo estoy muy lejos - de conocer, con suficiente precisión y detalle, cómo fue el desarrollo de esta nueva música en las catedrales portuguesas, así como en la capilla real.

En cambio, sí puedo asegurar que conozco, y he estudiado, un gran número de composiciones españolas de todo el siglo XVIII, así como del XVII, por lo que creo poder establecer ya, con plena garantía, consecuencias seguras y teorías del todo sólidas. Y creo deber insistir en que lo que se conoce de la música portuguesa lleva a la misma conclusión, ya que se ve con claridad que es idéntica a la española.

Pues bien: ese estudio me ha llevado a la convicción de que, sin minimizar, en modo alguno, el influjo de la música italiana, las numerosas composiciones de esta época, que se conservan en los archivos de casi todas las catedrales españolas, demuestran, con igual evidencia, que los compositores españoles, lejos de limitarse a copiar los modelos que les 
venían de fuera, crearon un estilo musical del todo propio, en el que, si bien son visibles los influjos foráneos, lo son igualmente los muchos elementos - algunos de los cuales son de los más determinantes para establecer un estilo musical - autóctonos, procedentes de la evolución interna de la música. Y de nuevo tengo que añadir que, por lo que se puede juzgar por lo publicado hasta ahora de la música religiosa portuguesa de este siglo XVIII, se puede asegurar para ella lo mismo que para la española.

Es notable que ya Kastner había llegado a la misma conclusión respecto a la música instrumental, y en concreto a las sonatas de tecla, en su magnífico estudio de 1988. Merece la pena copiar sus palabras:

"La polifacetada sonata bipartida era conocida y practicada en España y Portugal mucho antes de que Domenico Scarlatti llegara a Lisboa, Sevilla y Madrid. Es un error craso admitir que el Napolitano hubiera implantado este género en la Península Ibérica. No cabe la menor duda de que Domenico Scarlatti era uno de los muchos insignes maestros y tañedores de tecla de nuestra Europa occidental que practicaron asiduamente esta forma. Hízolo con ingenio invulgar, con su personalidad extremadamente fuerte y marcada. Ello no obstante, salvo algunos destellos de su impar técnica virtuosa, nada aportó que pudiese contribuir para el desarrollo en España y Portugal de la hechura de la galana sonata bipartida".

A continuación cita sendos párrafos de dos musicólogos españoles que para entonces habían publicado sonatas y otras composiciones de tecla de esta época en España: José Climent, que editó las de Vicente Rodríguez Monllor, y Román Escalas, que publicó las de José de Nebra, que habían llegado a una conclusión parecida, terminando con esta frase conclusiva:

"Huelga decir que las palabras de los señores Climent y Escalas no se refieren exclusivamente a España, sino también a Portugal; y no olvidemos que todavía en el siglo XVIII las relaciones y los intercambios musicales entre ambas naciones ibéricas eran muy intensas, lo que me lleva a hablar de preferencia de música ibérica"(2).

(2) Macario Santiago Kastner: "Carlos Scixas. Sus inquietudes entre lo barroco y lo prerromántico”, en Anuario Musical, 43, 1988, 163-187; las dos citas copiadas están en las págs. 166-167. 
Lo que acabo de decir respecto a la transformación de la música hispánica se refiere directamente a la música vocal, que sería más propio llamar sinfónico-vocal, a causa de la importancia que a partir de 1700 adquirieron los instrumentos.

En cambio, la de órgano tardó algo más en abandonar las formas que venían usándose, y no fue sino a partir de hacia 1730 cuando los cambios comienzan a ser visibles, también en este campo.

Vamos, pues, con este tema, que es, propiamente, el que me he propuesto estudiar en estas notas. Para mayor claridad dividiré mi exposición en dos partes, antes y después de Seixas, para, finalmente, tratar de hacer una síntesis o conclusión de lo que se deduce de los datos de que disponemos actualmente ${ }^{(3)}$.

Pero antes debo hacer una observación, que juzgo fundamental para comprender todo el proceso evolutivo de nuestra música, y en concreto el de la música de tecla: que el intercambio mutuo entre España y Portugal era entonces mucho más intenso de lo que es hoy. Buena prueba de ello son las varias - no sería exagerado calificarlas de numerosas - colecciones de composiciones organísticas que existen en Portugal, en las que la presencia de autores españoles es muy nutrida. Sin duda alguna, para aquellos organistas portugueses de los siglos XVII y comienzos del XVIII la música de los compositores españoles era idéntica a la que ellos mismos componían y tocaban. Es éste un dato que se debe tener siempre presente en todo este análisis que estamos haciendo.

Hay que partir de la situación de la música de tecla a fines del siglo XVII. Por consiguiente, no me ocuparé, por lo que respecta a Portugal, de los compositores de épocas anteriores, incluido el gran Rodrigues Coelho, y tomaré como punto de partida la obra del Padre Roque da Conceição. Su notable recopilación data, como es bien sabido, de 1695 e incluye obras de diversos compositores, siendo la mayoría de ellas anónimas, compuestas probablemente por el propio recopilador ${ }^{(4)}$.

(3) Ése cra mi propósito inicial. Pero, como digo al final de mi exposición, la extensión que alcanzó la primera parte me obliga a dejar la segunda para otra ocasión, que confío no esté lejana.

(t) Fr. Roque da Conceição: Livro de obras de orgao. Transcrição e estudo de Klaus Speer. Portugaliae Musica, vol. XI. Lisboa: Fundação Gulbenkian, 1967. 
Aun dejando a un lado los numerosos "versos", que por su misma naturaleza tenían una forma específica, independiente de todas las demás, importa analizar tres tipos de composiciones de las que él recopiló: los tientos, las obras y las fantasías.

Desde luego, una constatación se ofrece de inmediato; y se trata de una constatación importante: todas esas composiciones están concebidas exclusivamente para el órgano, más aún, para el órgano ibérico, tres de cuyas características principales eran la octava corta, el teclado partido y la falta de pedalier independiente. La primera de estas características se observa claramente en el dibujo que incluye al principio del volumen: un teclado de 42 notas, o sea, tres octavas no del todo completas más la octava corta; el teclado partido se exige en no pocos tientos de la colección; y la falta de pedalier independiente motiva el que él escriba habitualmente "a cuatro" y, por supuesto, sin una melodía propia para el pedal.

Todo esto es perfectamente sabido, pues era universal en todos los órganos ibéricos del siglo XVII. Pero era necesario recordarlo aquí, a fin de centrar adecuadamente la obra de Seixas y sus consecuencias.

Se puede afirmar, sin temor a errores ni exageraciones, que la forma fundamental, en el Padre Roque y en sus contemporáneos, era el tiento. El propio Kastner dedicó una atención especial a esta forma musical, siendo él quien primero abordó su estudio ${ }^{(5)}$. De hecho, las conclusiones que él expone en ese estudio siguen siendo válidas, y ese trabajo continúa siendo el punto de partida indispensable para el conocimiento de esta gran forma musical ibérica. Posteriormente se han ampliado diversos conceptos de los que él expone, y hoy podemos decir que tenemos una idea bastante exacta del tiento y su evolución. Aún quedan temas por dilucidar, y hay que decir que una de las fuentes que, a lo que me consta, está aún pendiente de ser objeto de un estudio monográfico es precisamente esta magnífica colección del Padre Roque, pues aunque ya Klaus Speer, en su introducción, hace un detenido análisis de los elementos de estos tientos, todavía queda mucho que decir en este punto. (Escribo esto con recelo, pues quizá haya habido ya sobre este tema alguna tesis doctoral, $\mathrm{u}$ otro trabajo similar, que no ha llegado a mi conocimiento; si esto fuera así, lo que es bien posible, pido excusas a mis colegas portugueses por ello).

(5) Santiago Kastner: "El tiento", cn su libro Contribución al estudio de la música española y portuguesa, Lisboa: Editorial Ática, Lda., 1941, págs. 145-192. 
Los tientos son, casi se podía decir que por esencia, contrapuntísticos, al menos los más extendidos. También los de Roque da Conceição son esencialmente contrapuntísticos. Pero yo noto una diferencia importante entre los tientos del compositor portugués - supuesto que los que no tienen nombre de autor sean de él; o aunque sean de otros compositores, pues eso es indiferente para su sentido histórico, y también para su significado artístico, - y los de sus coetáneos españoles, en concreto los de Cabanillas, que es el más prolífico compositor de tientos en la España de finales del siglo XVII y primeros años del XVIII. Frente al monotematismo de los del compositor valenciano, yo encuentro en los de la colección porteña una característica notable, que juzgo debe tenerse presente para comprender lo que vino luego, con Seixas y sus seguidores. Y es que fuera de la primera parte de la composición, en que por lo general expone un tema de contextura clásica en los tientos, que consiste en comenzar por una nota larga, generalmente una redonda, seguida por otras de valor algo más breve, generalmente algunas blancas, para ir reduciendo los valores rítmicos, fuera de esta primera parte, digo, el Padre Roque utiliza, sí, constantemente el sistema contrapuntístico como fundamento de la composición, pero a base de otros temas diversos, que reaparecen una y otra vez, incluso en diversas partes de la composición.

Lo más notable es que este mismo procedimiento compositivo se encuentra incluso en otro de los géneros musicales que él usa, aparentemente del todo alejado del tiento, cual es la fantasía. Es verdad que en esta forma suele comenzar con una música de carácter brillante, de improvisación melódica, con valores rítmicos reducidos. Pero luego vuelve, una y otra vez, a lo que, con toda evidencia, era su manera más propia de componer: la imitación contrapuntística. Presento, a simple modo de ejemplo, los compases 54-69 de su "Fantasía de 4..$^{\circ}$ tono" (Speer, págs. 53-54), donde juega, de modo extremadamente hábil, con dos temas simultáneos, que más que temas debieran ser llamados ideas musicales. No llega, naturalmente, a someterlos a un desarrollo temático estrictamente dicho, pues era éste un concepto para cuya estructuración faltaban casi cien años, pero sí usa una técnica que se le parece no poco. 


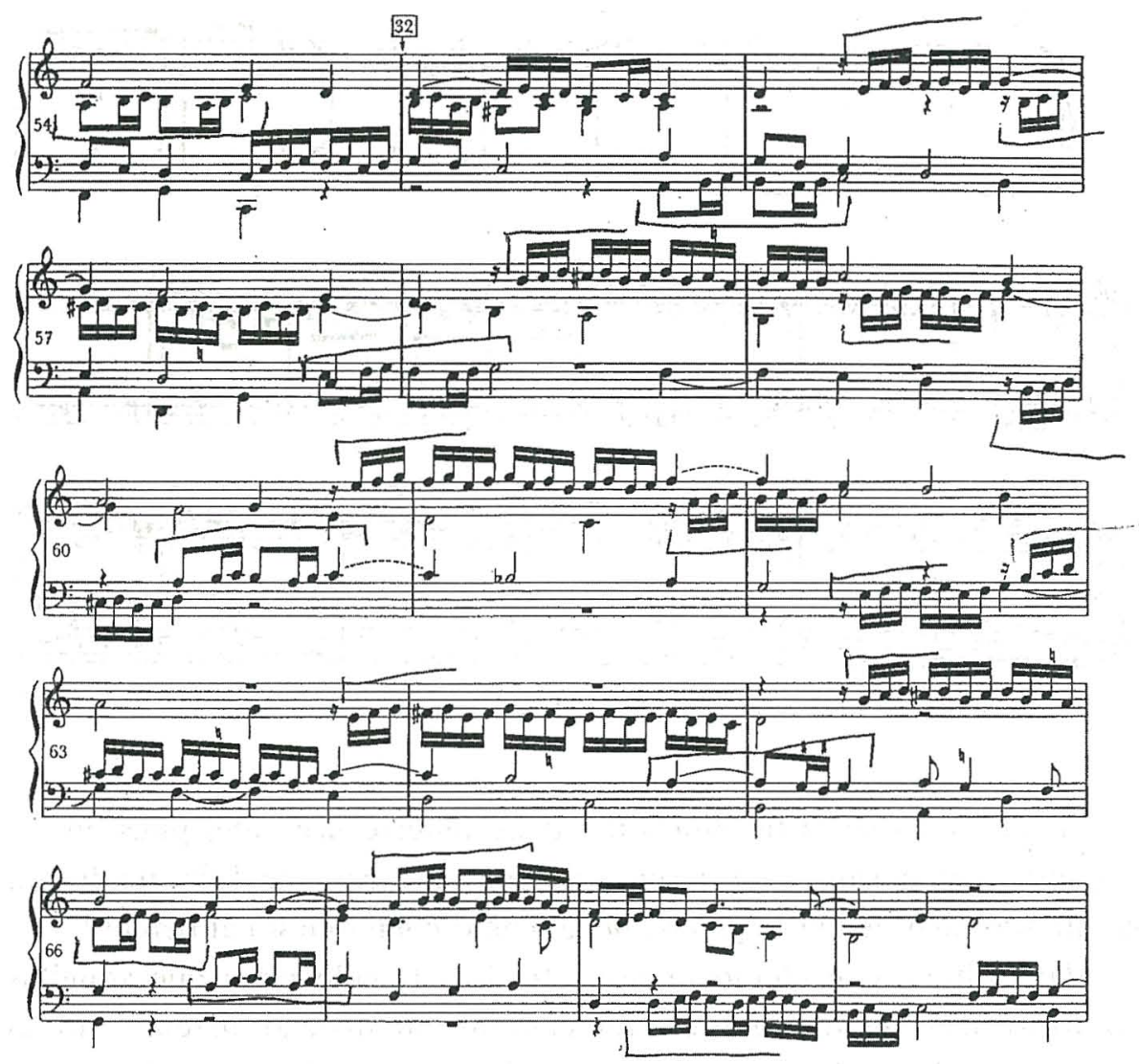

Ej. 1. Roque da Conceição (?): Fantasia de $4 .^{\circ}$ Tom.

En realidad, la técnica de la imitación contrapuntística la tenían tan metida en la sangre nuestros organistas de la segunda mitad del siglo XVII, que, sin salirnos de la colección de Roque da Conceição, la usa incluso en el comienzo mismo de algunas de sus fantasías, por ejemplo ya en la primera que publica, la "Fantasía de 1. ${ }^{\circ}$ Tom", que, como se ve, es un verdadero "tiento".

Lo mismo hace en las "Obras", hasta el punto de que resulta difícil diferenciarlas dé los tientos. Ya Speer lo notó cuando escribe, en la página XVI de la introducción:

"Obra parece ter sido um termo empregado pelo amanuense que escreveu o índice para abranger tentos e phantasias, dois termos que podiam ter sido usados alternadamente, ambos dizendo respeito à forma da composição". 

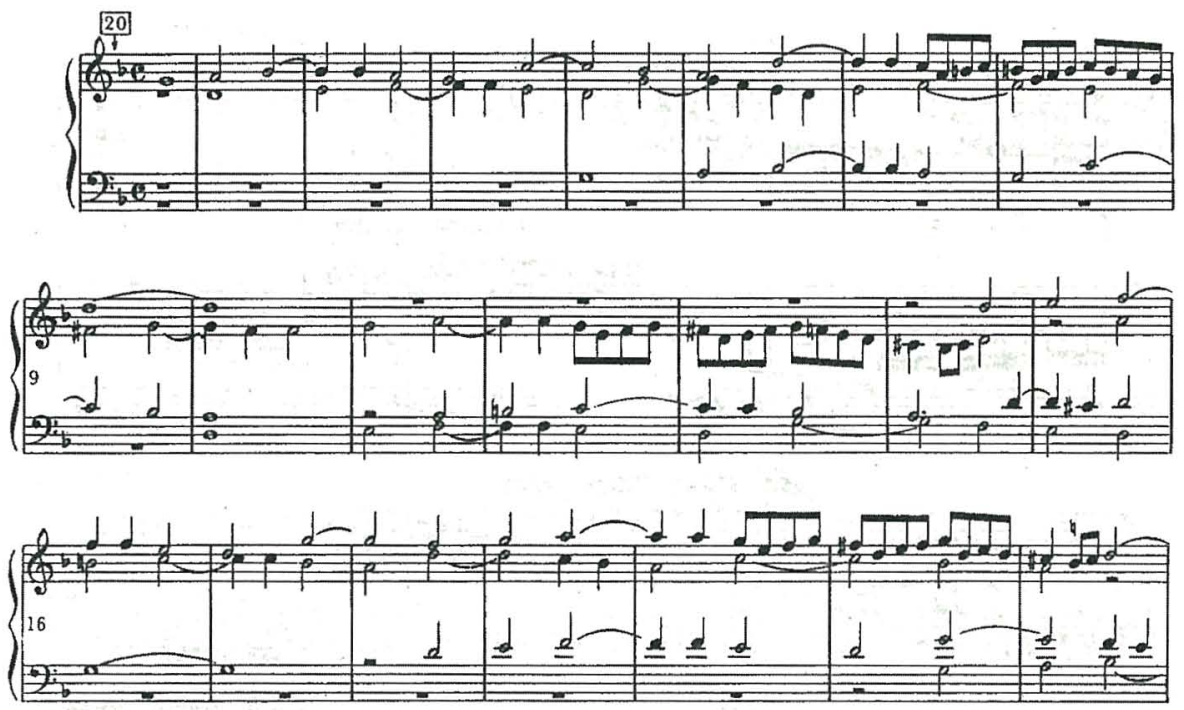

Ej. 2. Roque da Conccição (?): Fantasia de $10^{\circ}$ Tom.

Y sin embargo el término obra debe tenerse presente, pues en los organistas-compositores de después de aproximadamente 1720 tendrá un significado bien definido, precisamente como contrapuesto al tiento ${ }^{(6)}$.

Una última consideración respecto de esta colección: que aquellos compositores seguían atados, férreamente atados, al sistema modal eclesiástico, de modo que no solamente los tientos y las "obras" - y, por supuesto, los versos, aunque en éstos el sistema "modal", o "tonal", era poco menos que obligatorio y necesario, para poder alternarse con el canto llano del coro -, sino incluso en las fantasías: también éstas están compuestas, en esta colección de Porto, cada una en un determinado "tono" eclesiástico.

Pero son muy hermosas e interesantes. Y es una gran pena que no sean más conocidas e interpretadas, pues no cabe duda que constituyen un hito importante en el desarrollo de las formas organísticas de nuestra península.

Y aún añadiría una observación más, que juzgo importante. Frente a la opinión de Speer (introducción, pág. VIII), de que esta colección había sido

(6) Es ése, como se ve, un concepto contrario al expresado por Speer. Pero es que cuando él escribió eso no se conocían los muchos datos que aparecieron después, que demuestran que el término obra tenía para los organistas ibéricos de finales del siglo XVII y primer cuarto del XVIII un significado preciso, diverso del tiento y de la fantasía. 
recopilada para uso individual de un tañedor de tecla, o para su escuela, a mí me da la impresión de que la idea del Padre Roque en acometerla fue para publicarla. Es verdad que faltan en el manuscrito las habituales aprobaciones que figuran en las colecciones de aquel tiempo publicadas o destinadas a la publicación. Pero el conjunto, y en particular algunos detalles de él, sugieren más bien esa finalidad concreta, de la publicación. Que luego alguien añadiera alguna composición, o grupo de composiciones, a la colección original - si es que, como supone Speer, la colección no es autógrafa del Padre Roque, lo que no es del todo seguro, - no cambia la esencia de lo que debió de ser la idea del compilador. El modo mismo como está concebido y redactado el índice, y sobre todo detalles como el del dibujo del teclado al comienzo, la fecha de la portada, etc., todo hace suponer que él, con su iniciativa, pretendía algo más que reunir un simple conjunto de obras para su uso individual. No suele hacerse una orla tan hermosa y cuidada para enmarcar el título o el teclado del órgano, con todo aquel detalle de cada tecla en el sistema de las escalas guidonianas, para tan sólo ponerlo en el atril del órgano y tocar por él.

No resulta fácil seguir la evolución de la música portuguesa de órgano entre Roque da Conceição y Carlos Seixas. Ni siquiera parece que existan actualmente obras que se pueda asegurar que sean de estos años, al menos en cantidad suficiente como para poder ir sobre seguro.

En cambio, las hay en España, y en abundancia. Cierto, no se puede deducir solamente por ese hecho que lo mismo haya sucedido en Portugal, pues ni siquiera en España circulaban demasiado de una catedral a otra. Y a pesar de eso creo que se puede aplicar lo mismo a Portugal, ya que el hecho incuestionable es que el desarrollo de las formas musicales, en las organísticas como en las vocales, es idéntico, y simultáneo, en ambas naciones. Más aún: hay organistas - Francisco Andréu, Andrés de Sola, Cristóbal Brocarte... - que tienen obras que, aunque compuestas para órgano, tienen ya mucho de clavecinísticas y demuestran que el lenguaje clavecinístico no sólo no les era desconocido, sino que formaba ya parte de su modo de pensar la música. Y de nuevo hay que volver a la constatación que ya queda hecha: que precisamente estos tres autores, con otros coetáneos españoles, se encuentran abundantemente presentes en algunas de las colecciones portuguesas de composiciones para órgano de estos años. Séame permitido reproducir aquí un fragmento de un "tiento a tres" del primero 
de los autores citados, que ya publiqué en 1983 , en el volumen $3 .^{\circ}$ de la Historia de la Música Española de Alianza Editorial:
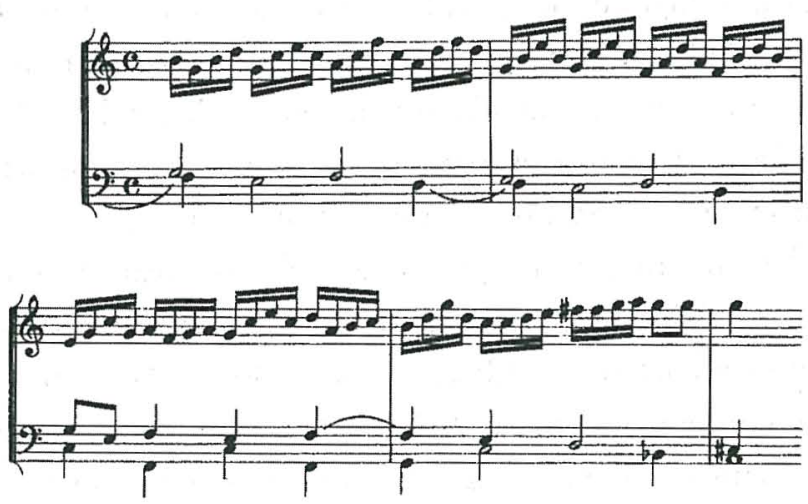

Ej. 3. Francisco Andréu: Tiento sobre el "Ave maris stella”.

Merece la pena que nos paremos un momento en un compositor particular, a causa del significado que él y su obra tienen en el desarrollo de las formas musicales para órgano en la península ibérica en torno a 1720: José Elías. Nacido hacia 1687 en Barcelona, fue, a partir de 1712, organista en varias parroquias de su ciudad natal, hasta que, en 1725, obtuvo igual cargo en el ambicionado puesto del convento de las Descalzas Reales de Madrid, cargo que parece conservó hasta su muerte, acaecida hacia 1755. Se conservan varias obras suyas datadas en sus años de Barcelona, y, según el testimonio del Padre Soler, algunas de las de esa época fueron copiadas también para el monasterio de Montserrat.

Su obra principal es la colección que recopiló mucho más tarde, en 1749, para la imprenta, cuyo título lo dice todo:

"Obras de órgano, entre el antiguo y moderno estilo. Cláusulas sonoras, que expresan la más dulce y suave armonía. Contienen doce piezas, las seis primeras patéticas, sin más intentos que tocarlas de paso, para cuando se alza a Su Divina Majestad; y las otras seis más vivas, con asuntos determinados, sobre los Cánticos de Nuestra Señora, para los ofertorios. Unas $\mathrm{y}$ otras desnudas de toda glosa y ornato correspondiente, y vestidas solamente de lo sustancial, así por lo conciso de ellas como por haberse escrito únicamente a fin de que los 
profesores principiantes de esta facultad tengan luz y guía para aprender a tocar suelto y seguir un paso por los términos conducentes del tono con la más perfecta y natural modulación".

Concorde con ese programa compositivo y estético está el contenido: la segunda serie de composiciones que ahí anuncia, los "ofertorios", constan todos de dos partes, generalmente marcadas por los tempos: Andante-Allegro, que se corresponden, en todo y por todo, con lo que los coetáneos germánicos llamaban "preludio y fuga". El "andante" es un auténtico "preludio", de carácter improvisatorio y brillante; los "allegros", por el contrario, no solamente están compuestos en riguroso estilo contrapuntístico, sino que son verdaderas "fugas", en el más estricto sentido, hasta con sus diversos elementos, partes constitutivas, etc.

Es decir: que entre 1725 y 1740 , o antes, cuando, presumiblemente, la estructura de la nueva forma musical estaba del todo configurada, se verificó, entre los compositores españoles de música para órgano, y, por lo que se ve de las obras actualmente conocidas, igualmente entre los portugueses, una transformación total del concepto de forma contrapuntística. Este hecho, en Elías, adquiere un significado del todo particular en cuanto que él se declara discípulo apasionado de Cabanillas, de cuyas obras confiesa que llegó a copiar de su mano, en su juventud, más de 300.

Dejo, por el momento, sacar las consecuencias de un hecho tan sorprendente, pues me parece preferible seguir con el desarrollo de las formas de la música de tecla en la Península, tomada en su acepción global, ya que algunas de las consideraciones que se ofrecen a propósito del binomio Elías-Cabanillas son las mismas que plantean otros autores ibéricos de estos años, y en concreto, por lo que hoy estamos considerando, Carlos Seixas respecto de sus inmediatos predecesores, y aun los compositores portugueses y españoles para música de tecla que vinieron después de él.

Vamos, pues, a tratar de este compositor más en detalle.

Sus principales datos biográficos son bien conocidos: José Antonio Carlos Vaz nació en Coimbra el 11 de junio de 1704. Músico precoz, a solos 14 años sucedió a su padre, que acababa de morir, como organista de la catedral de su ciudad natal. Dos años después - 1720 - se trasladó a Lisboa, donde obtuvo el puesto de organista de la capilla real y de la seo patriarcal, que conservó hasta su muerte, acaecida el 25 de agosto de 1742. En Lisboa 
cambió su apellido por el de Seixas, sin que se sepa exactamente el porqué; la opinión más verosímil es la suposición de Kastner y otros: que lo hizo en reconocimiento al noble brasileño João de Seixas da Fonseca Borges, que fue quien propició la publicación del volumen de Sonatas de Ludovico Giustini, de que vamos a hablar inmediatamente ${ }^{(7)}$.

Este compositor italiano, el primero en publicar un volumen de composiciones específicamente para el recientemente inventado piano-forte, nació en Pistoia en 1685 y murió en la misma ciudad en 1743. En 1732 publicó en Florencia su "opus 1": un volumen que tituló 12 Sonate da cimbalo di piano e forte, detto volgarmente di marteletti. Ese libro tiene una doble circunstancia que importa mucho para el tema que estamos estudiando: que está dedicado al Infante de Portugal, don Antonio, hermano del rey don Juan V, y que la dedicatoria no la firma el compositor, sino el citado caballero brasileño, don Juan de Seixas. Los problemas que plantea esta colección de sonatas, entre ellos el de cómo y por qué el Infante don Antonio las oyó, posiblemente en Florencia, por qué firma él la dedicatoria, y precisamente al rey de Portugal, dónde se imprimió el libro, por qué no existen copias de él en Italia - tan sólo una - y sí en Portugal y otras naciones europeas..., ésos, y varios otros, los analiza detenidamente Freeman en su exhaustivo estudio de $2003^{(8)}$.

Aquí es suficiente recordar tres hechos: que ese libro fue publicado en 1732; que, como se acaba de decir, es el primero - "con mucho", añade Freeman (pág. 111) - en escribir específicamente para el piano-forte; y que se puede dar como seguro que Seixas lo conoció. Del influjo que haya ejercido en Seixas y en su concepción de la sonata de tecla hablaré en seguida. Antes hay que tocar el tema del otro italiano compositor de sonatas que tuvo que ver con Seixas: Domenico Scarlatti.

(7) A los datos que ya conoció Kastner cuando escribió su biografía de Seixas en 1947 sobre este personaje, que llegó a obispo, se añaden los encontrados por Robert Stevenson y publicados en 1968 (Robert Stevenson: "Some Portuguese Sources for Early Brazilian Music History", en Yearbook of the Inter-American Institute for Musical Research, 4, 1968, págs. 2-6), que incluyen varios puntos fundamentales de su biografía, aclarando, de modo poco menos que definitivo, el porqué de su relación con Giustini e indirectamente con Carlos Vaz-Seixas. El propio Kastner los utilizó, sacando las importantes consecuencias que de ellos se derivan, en el artículo de 1988 (“Carlos Seixas. Sus inquietudes...”, págs. 168-169).

(8) Danicl E. Freeman: "Lodovico Giustini and the emergence of the keyboard sonata in Italy", Anuario Musical, 58, 2003, 111-138. 
No estará de más recordar que este famoso músico comenzó su carrera, no como organista, y menos como compositor de sonatas de tecla, sino como maestro de capilla, y nada menos que de la Cappella Giulia de San Pedro de Roma, cargo para el que fue nombrado en 1714 - tenía entonces 29 años y llevaba ya más de uno de ayudante del maestro -, y en el que siguió hasta 1719, en que se trasladó a Lisboa, a donde llegó el 29 de noviembre, de nuevo no para un empleo ligado a los instrumentos de tecla, y precisamente de los de cámara - clavicordio o clavecín -, sino como maestro de la capilla real. Por tanto, su misión primordial en Lisboa era la de componer música para la capilla real y patriarcal, y dirigir las funciones solemnes. Es verdad que hay buenos motivos para pensar que la opinión generalizada, de que el rey le llamó a sugerencia de su hermano, el Infante don Antonio, quien parece que conoció a Scarlatti en Roma en 1714 y que fue quien sugirió al soberano que ése podría ser un magnífico maestro de música de la Infanta María Bárbara, responde a la realidad de los hechos; pero los documentos descubiertos por Doderer, que tanta luz arrojan sobre la estancia de Scarlatti en Lisboa ${ }^{(9)}$, confirman lo que ya se sabía en base a otros documentos: que Scarlatti vino a Lisboa como maestro de capilla y que, efectivamente, ése fue su primer empleo en la capital portuguesa, con todas las consecuencias que llevaba consigo. Eso sí: fue recibido con grandísimos honores, gozando siempre de la estima de los soberanos y llegando al extremo de que alguna vez cantó ante la corte acompañándole al clave la reina en persona.

Eso fue a los comienzos. Pero pronto asumió los nuevos encargos, de ser el maestro de música del Infante don Antonio y sobre todo de la Infanta doña María Bárbara, acabando por asumir este último empleo casi a tiempo completo y componiendo para ella muchas de sus numerosísimas sonatas (pero todo hace suponer que algunas, quizá varias decenas de ellas, las tenía ya compuestas antes de venir a Portugal). Y cuando la Infanta se desposó con el Príncipe español don Fernando, el 19 de enero de 1729, Scarlatti siguió a su regia alumna a Madrid, que desde entonces se convirtió en la ciudad de su residencia y donde moriría en 1757.

(9) Gerhard Doderer: "Aspectos novos em torno da esadia de Domenico Scarlatti na corte de D. João V (1719-1727)”, prefacio a Domenico Scarlatti: Libro di toccate per cembalo, Lisboa, 1991. 
Todo eso es ya sabido, pero era necesario recordarlo aquí para entrar con pie seguro en el estudio histórico que deseo hacer. Más aún: creo que será bueno resumir los hechos principales y sus fechas, para proceder con más seguridad:

1719. El 29 de noviembre llega Domenico Scarlatti para hacerse cargo del magisterio de capilla de la capilla real y de la seo patriarcal; sigue en Lisboa hasta enero de 1729, en que, tras la boda de María Bárbara con el Príncipe Fernando de España, el futuro Fernando VI, pasa a vivir a Madrid.

1720. Llega Seixas (Vaz) a Lisboa; es nombrado organista de la capilla real y de la Seo Patriarcal; mantiene ese cargo hasta su muerte en 1742.

Es decir: Entre 1720 y 1729 Seixas y Scarlatti no solamente conviven en una misma ciudad, Lisboa, sino que sus respectivos cargos, de organista y maestro de capilla de la misma institución, hacían que su trato fuese, más que frecuente, diario.

1732. Se publican las doce sonatas de Ludovico Giustini.

Las ochenta sonatas que se conservan de Seixas - otras muchas se han perdido, seguramente que en el terremoto de Lisboa de 1755 , - se conservan manuscritas y han sido publicadas en su integridad por Kastner (Portugaliae Musica, X), en cinco fascículos: uno de introducción y cuatro de edición; además, hay otras muchas ediciones parciales, a cargo del propio Kastner, antes de esa edición completá, y por otros autores. En los análisis y comentarios siguientes sigo el orden de la edición completa de Kastner.

Bien. Es llegado el momento de resumir en unas pocas frases el significado de todos esos hechos históricos que acabamos de enumerar.

Soy consciente de la responsabilidad que implica emitir una opinión personal en un tema en el que ya tantos otros autores han entrado, sobre todo porque mis opiniones no siempre coinciden con lo que otros han escrito. No obstante el miedo que causa esa responsabilidad lo voy a hacer con plena determinación. Quizá tenga como justificación el hecho de que lo que les voy a decir sintetiza mis conclusiones después de varios años más de veinte - estudiando este tema, de la música de tecla en la península ibérica en el siglo XVIII. De hecho, cuando el coordinador del presente coloquio, Dr. Pedrosa, me propuso, en el mes de febrero pasado, tomar parte en él, yo le respondí, al aceptar, que lo hacía porque ello me permitiría poner un poco en orden mis ideas acerca de este tema, que he tratado en diversos cursos y otras ocasiones, pero sobre el que nunca me he atrevido a publicar nada, porque no veía claros importantes detalles de él. 
Resumo, pues, mis conclusiones en los siguientes puntos:

1. Parece claro que la idea de "sonata" instrumental la recibió Seixas de Scarlatti, quien, además de las sonatas que eventualmente hubiera traído de Roma en 1719, se puede dar por seguro que entre esa fecha y 1729 compuso un buen número de ellas para sus dos discípulos regios, sobre todo para María Bárbara ${ }^{(10)}$.

Esto se deduce del hecho de que antes de esa fecha no parece que se conociera el concepto de sonata instrumental, al menos para instrumentos de tecla, en Portugal ni en España ${ }^{(11)}$. Al menos, a lo que yo recuerdo, no se encuentra ese nombre en ninguna composición anterior. Ni era fácil que tal concepto entrara, pues los compositores anteriores para tecla eran todos organistas y componían para órgano, y precisamente dentro de unos parámetros, unos criterios, unos ambientes concretos, determinados, que no dejaban mucho margen para innovaciones.

2. No parece que pueda dudarse tampoco de que la obra de Giustini, conocida en Lisboa a partir de 1732, ejerció un poderoso influjo sobre Seixas.

Esto se deduce del hecho de que algunas de las sonatas que Seixas compuso se parecen más a las de Giustini que a las de Scarlatti.

3. Pero las sonatas de Seixas no son, en modo alguno, simples imitaciones de las de los dos compositores italianos. Es éste un concepto repetidas veces expuesto por Kastner ${ }^{(12)}$. Sólo que él nunca se paró a demostrar, diríamos que gráficamente, con ejemplos musicales convincentes, del porqué de su aserto.

(10) Esta opinión mía se contradice con la de Kastner, que ya queda copiada, de que la sonata bipartita era conocida por los compositores ibéricos antes de la llegada de Domenico Scarlatti a la Península. Me cuesta disentir de quien, por tantos conceptos, tengo por mi maestro. Pero la verdad es que no conozco un solo ejemplo que avale la opinión de Kastner. Otra cosa muy distinta es lo que Scixas y otros compositores ibéricos hicieron con la forma musical que, a mi juicio, recieron de Scarlatti, y de que hablaré inmediatamente.

(11) La única "sonata" anterior a csas fechas que se conoce en la Península Ibérica es la "sonata a tres" de José de Vaquedano, compuesta en Santiago de Compostela, en torno al año 1695 ; no es, pues, sonata de tecla.

(12) Kaster incluso tiene expresado, y varias veces, este concepto, de la diferente concepción de la sonata de tecla, no sólo en Seixas y en Scarlatti, sino, en modo más genérico, por parte de los compositores de tecla ibéricos y sus coetáneos italianos. Así, por tejemplo, hablando de Correa de Araujo (Carlos Seixas, pág. 43), escribe:

"A comparação das obras dos contemporános ibéricos de Pasquini com as deste [Correa] evidenciará a diferença da evolução da música de tecla havida na Península e na Itália”. 
Creo que es llegado el momento de hacerlo, por lo mucho que nos interesa. Por eso me paré tanto en hablar de lo que era la música de tecla en la Península al momento de asumir Seixas el puesto de Lisboa, pues sólo así, comparando su música con la de sus predecesores portugueses y españoles, y con la de los dos compositores italianos que influyeron en él, se puede sostener con seguridad esa afirmación.

Seixas toma, sí, de Scarlatti y de Giustini el nombre de sus composiciones, es decir, la "sonata", quizá incluso el concepto genérico, y hasta, si se quiere, la forma musical, al menos en su estructura fundamental; pero la música que él compone no proviene de ellos, sino de su propia inspiración, condicionada, de modo determinante, por la tradición ibérica. De tal modo, que se puede afirmar, con toda seguridad, que las sonatas de Seixas, lo mismo que las de los compositores que le siguieron, tanto en Portugal como en España, siguen una orientación propia, creada por ellos, en base a la tradición musical ibérica, y llegan a constituir una forma musical del todo peculiar, diferente - no mejor ni peor; simplemente diferente - de las homónimas de otras naciones.

¿Cuáles son, pues, estas características que diferencian las sonatas de Seixas de las de Scarlatti y Giustini? He aquí algunas:

1. Forma musical. Scarlatti tiene un concepto de sonata único, que mantiene intacto en prácticamente toda su inmensa producción, con tan pocas excepciones, que no invalidan para nada la regla general. Es la sonata bipartita, con la segunda parte tomada de la primera en la dominante o en otra tonalidad cercana y con pocas variaciones.

Seixas, en cambio, aunque conoce, y a veces usa, ese tipo de sonata, tiene de la forma un concepto mucho más amplio. Lo primero que salta a la vista es que frente a la concepción scarlattiana de componer sus sonatas en un solo tiempo en dos partes, que, como ya queda dicho, es única y la usa en todas sus sonatas, Seixas compone una gran parte de las suyas en dos, y hasta en tres y cuatro tiempos. Estos segundos tiempos de las sonatas seixanas suelen ser un minueto; pero también las tiene con dos minuetos, o incluso con otras variedades de segundos o terceros tiempos, llegando al extremo de la sonata 49, en sol menor, en que después del allegro inicial tiene un "adagio", al que sigue un "andantino", para terminar en un "amoroso". 
2. La música misma. Scarlatti mantiene, en esencia, un único lenguaje musical para sus sonatas, que fluye como espontáneamente, siempre con gran brillantez, pero sin elementos internos que mantengan, de alguna manera, la unidad del discurso musical, por ejemplo con motivos que se repitan, transformaciones de otros, etc. El todo da la impresión de una vena melódica extraordinaria, con una inspiración inagotable, que se mantiene siempre fiel a sí misma, pero que, no obstante esa persistencia, resulta siempre nueva. Tiene, por supuesto, a veces algunos elementos que reaparecen en el devenir del discurso musical, tiene incluso sonatas concebidas en plan imitativo, pero se trata de excepciones casi aisladas, que no invalidan la norma general.

Seixas, en cambio, usa mucho, y de forma consecuente, otros tipos de composición musical, además del brillante de tipo "fantasía", que también tiene. Por ejemplo, la imitación, que se ve claramente que en él proviene de la tradición ibérica de la música de órgano del siglo XVII. Hay ejemplos notabilísimos de esto. Por ejemplo, la sonata 71, en la menor, donde también se percibe un segundo principio compositivo, que no existe en Scarlatti salvo casos aislados: el de usar fragmentos temáticos, ideas musicales, que repite y transforma, convirtiéndolos casi en unos "temas", que se acercan mucho al desarrollo temático:
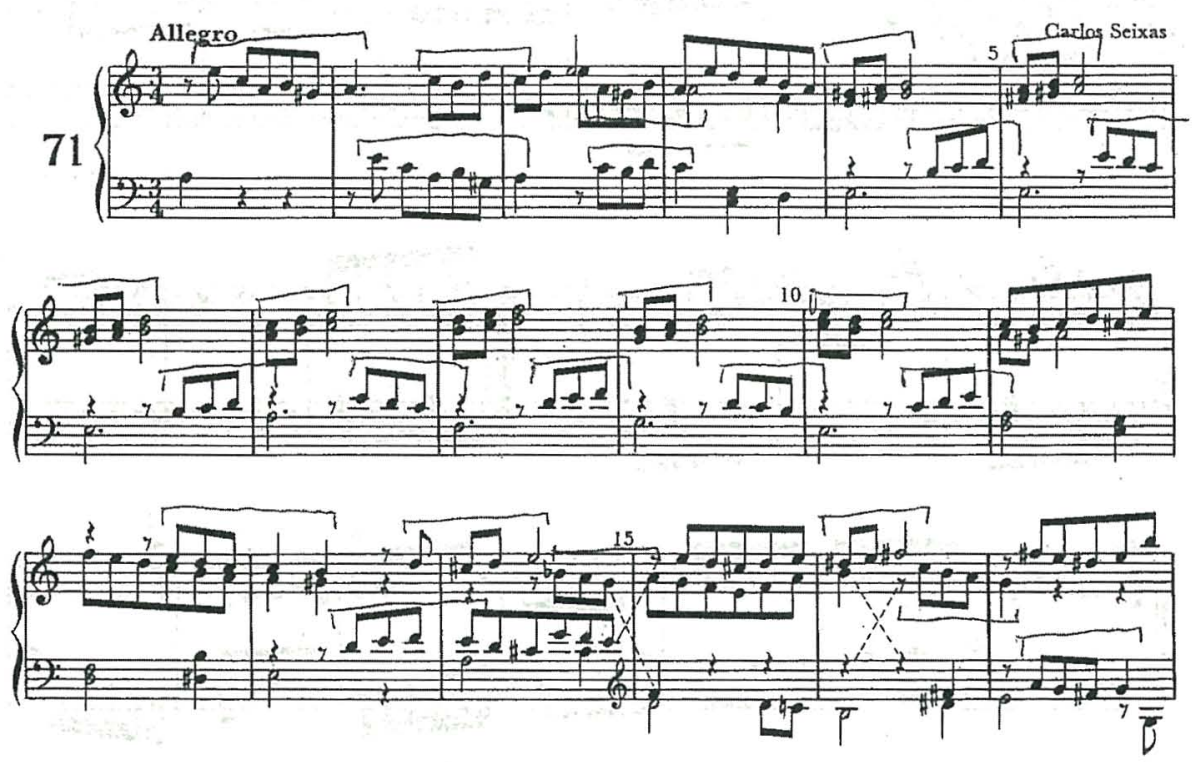

Ej. 4. Carlos Seixas: Sonata 71, en la menor. 
Es evidente que estamos ante una composición hecha, no como una melodía continuada, al estilo de Scarlatti, sino en base a ideas musicales que se repiten, se alternan, dialogan entre sí, se transforman. El parentesco entre esta concepción de la composición musical y la de Roque da Conceição es clarísimo.

3. Frente a la concepción scarlattiana de que la segunda parte de la sonata repitiera la primera a la dominante o a otra tonalidad cercana, pero sin variar sustancialmente los motivos melódicos o rítmicos, Seixas, aunque conoce y practica este sistema compositivo, usa de otros muchos medios, por ejemplo no repitiendo el primer tiempo en el segundo, sino componiendo éste en un modo diferente; quizás a base de elementos del primero, pero cambiándolos incluso totalmente o casi. Los ejemplos se podrían multiplicar en gran número. He aquí uno, que sirva por muchos: la sonata 47 en sol mayor:
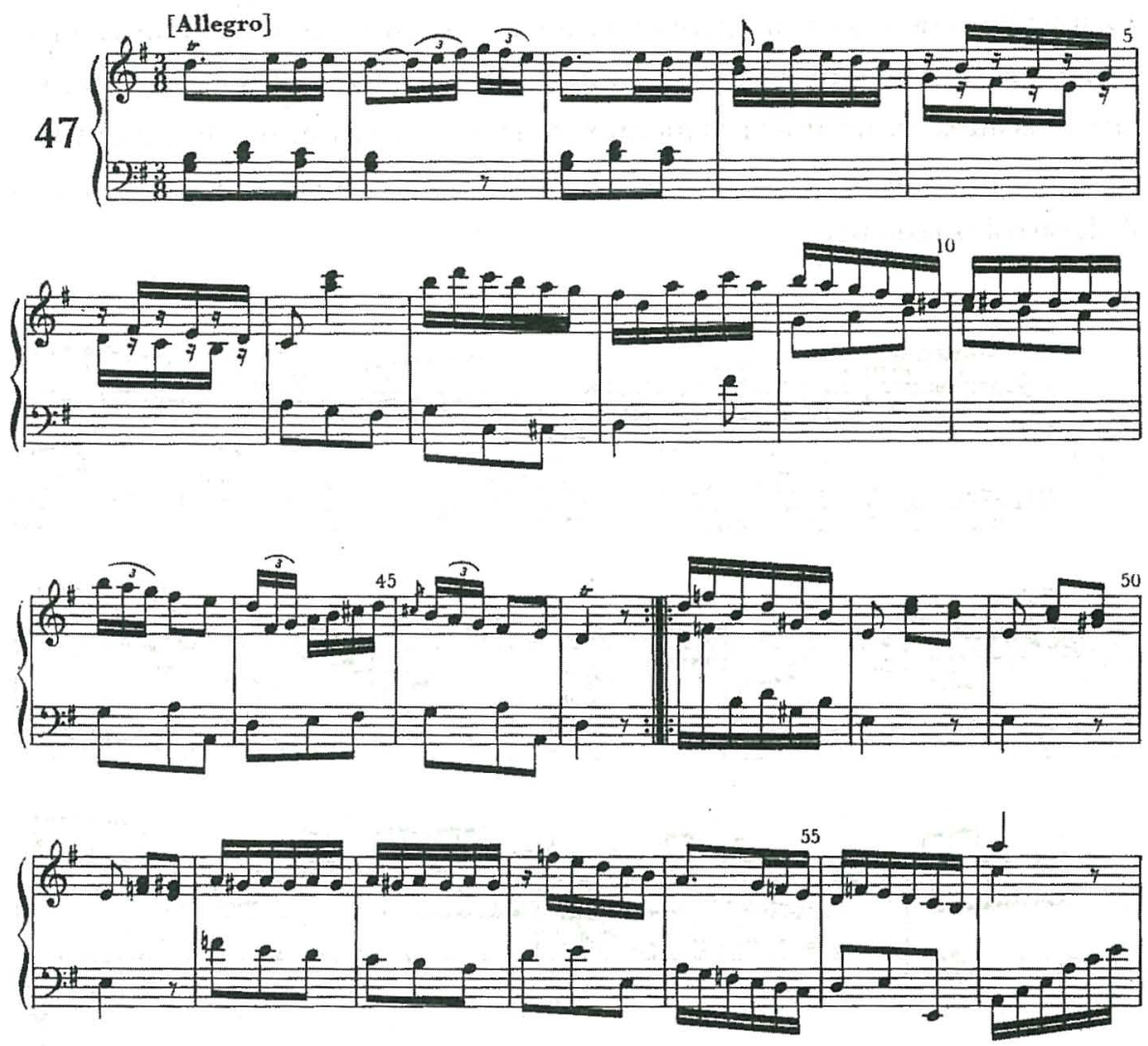

Ej. 5. Carlos Seixas: Sonata 47, en sol mayor. Comienzo de las partes primera y segunda. 
4. Scarlatti concibe sus sonatas - todas, sin una sola excepción, - para el clavecín. Esto se deduce con toda claridad de la música misma. Seixas, aunque muchas de sus sonatas sean también perfectamente aptas para ser interpretadas en el clavecín, en otras se ve que las compuso pensando en el clavicordio; más aún: algunas parecen pensadas para el piano-forte ${ }^{(13)}$. Piénsese, por ejemplo, en las manos cruzadas de la sonata 19 , o en el segundo minueto de la sonata 42 , pasajes ambos - y hay otros muchos similares - que no parece que se puedan interpretar adecuadamente a no ser en un instrumento que permita relizar el "forte" y el "piano", e incluso, y sobre todo, mantener la sonoridad de los acordes arpegiados a través del pedal que ásle los macillos ${ }^{(14)}$. Véase el comienzo de ese segundo ejemplo citado, juntamente con el minueto primero, del que el segundo es, como bien observa Kastner en su anotación, una "glosa" del precedente; o sea: un ejemplo más de la continuidad de Seixas respecto de la música portuguesa, e ibérica en general, inmediatamente precedente.

(13) Doderer, en su libro Clavicórdios portugueses do Século Dezoito (Lisboa: Fundação Gulbenkian, 1971), describe con gran detalle los quince clavicordios que se conservan en el museo del Conservatorio Nacional de Lisboa, que son una muestra magnífica de la difusión que este instrumento tuvo en Portugal en este siglo XVIII que estamos estudiando.

Nada dice Doderer del piano-forte, ni conozco información precisa de los instrumentos de este tipo que pudo haber en Lisboa en los tiempos de Seixas, aunque se puede dar por seguro que los había.

(14) Durante el simposio se nos ofrecieron, a los asistentes al mismo, y al público en general, tres conciertos con sonatas de Scixas interpretadas en el órgano, en el clavecín y en el piano. Hubo opiniones diversas entre los asistentes acerca de lo que se podría definir como la "validez" de algunas de esas tres interpretaciones, es decir, respecto del instrumento en que se interpretaban, en particular el piano.

Si me es lícito expresar mi propia opinión, deberé decir que a mí me convencieron las tres, en un grado aproximadamente igual. Cierto que el sentido que adquirían las composiciones - varias de las sonatas se interpretaron en los tres instrumentos - era no poco diverso, según el instrumento en que se las oía; pero la "validez", la "autenticidad", me parecieron idénticas, o casi, en las tres. Es claro que el "piano" que conoció Seixas, y para el que, según hoy podemos deducir, compuso algunas de sus sonatas, era muy diverso del actual, eso desde luego; pero no cabe duda que si él hubicra conocido los pianos de hoy no habría dudado en tocarlas en ellos. Que si hubiese conocido el piano de hoy habría escrito sonatas bien diversas no parece que pueda ponerse en duda. Pero eso no invalida el que sea válido y auténtico tocar las que compuso, y como las compuso, en el piano moderno. De hecho, cl Maestro Martíns, que fue quien las interpretó al piano en cl tercero de aquellos conciertos, tiene un buen número de ellas grabadas en el piano y publicadas en varios discos, en unas versiones que me parecen perfectamente aceptables, Salvo, claro está, meliori iudicio..., es decir, salva siempre la opinión contraria, que es claro que la hay y que es, o puede ser, tan válida o más que la mía. 
MINUET

[Espressivo]
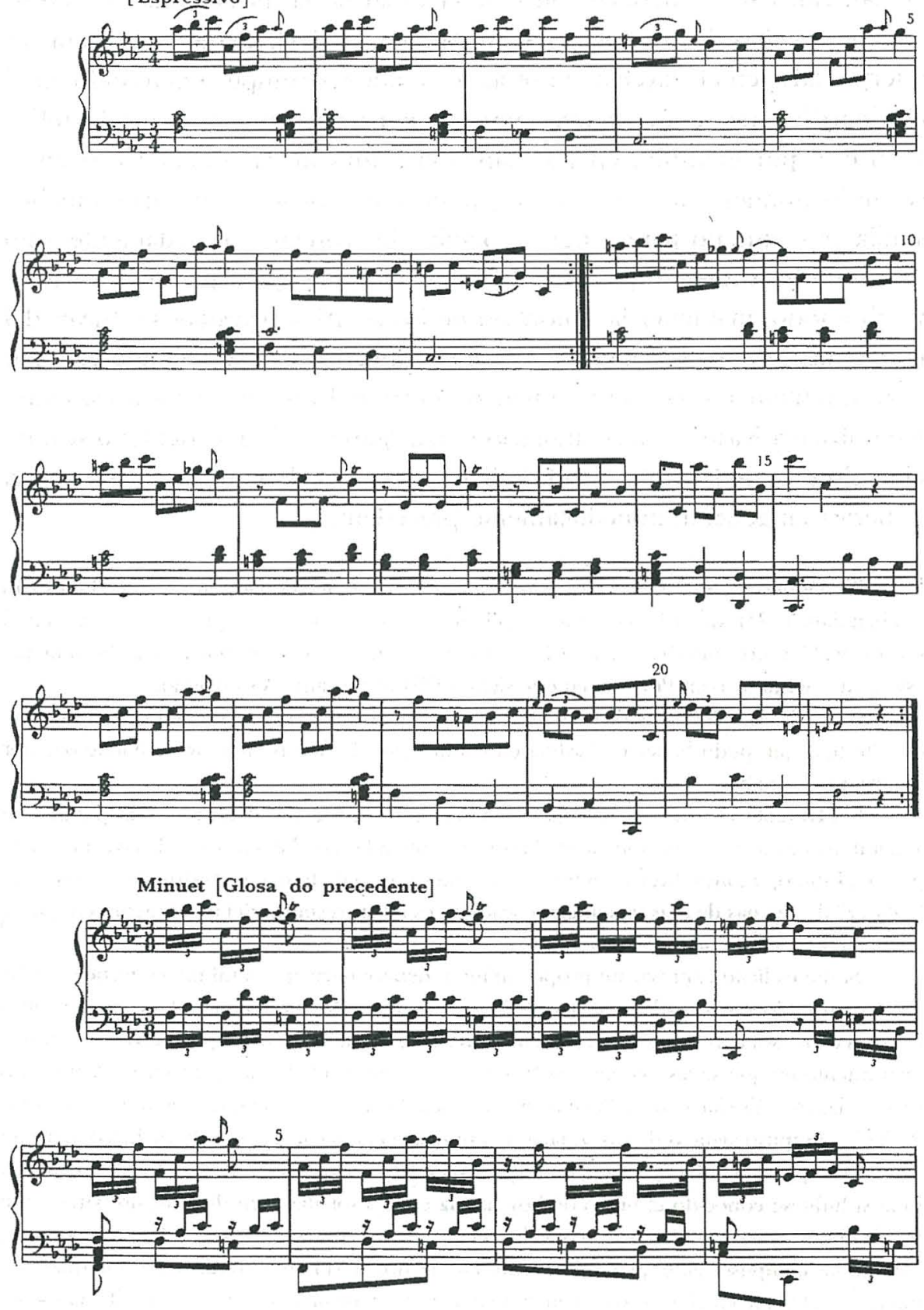

Ej. 6. Carlos Seixas: Sonata 42, en fa menor. $2 .^{\circ}$ movimiento. 
Creo, pues, que está perfectamente justificada la opinión expuesta, que es la misma conclusión a la que había llegado Kastner: que, aun admitiendo que el punto de partida de Seixas haya sido Scarlatti, el maestro portugués, lejos de limitarse a copiar a su modelo, o, simplemente, a seguir por el mismo camino, creó un estilo musical del todo nuevo, que tiene mucho de lo anterior de su país y mucho, sin duda, también de su propio genio personal, y que se podría resumir así: Seixas conoció la sonata de tecla, en su concepción y en su forma musical, de Scarlatti, pero no la copió sin más, en su forma ni menos en su lenguaje, sino que en ambos conceptos, y sobre todo en el segundo, el lenguaje musical, supo encuadrar ese concepto genérico en la música que era la suya, la portuguesa, y aun ibérica en general, creando, en realidad, una forma musical propia, prácticamente nueva, en su concepción y en su desarrollo, y sobre todo utilizando para esa nueva forma musical, verdaderamente ibérica, el lenguaje musical heredado de sus predecesores, aunque transformado por él $\mathrm{l}^{(15)}$.

La comparación con Giustini es más fácil y tiene menos elementos. Es verdad que Seixas vivió, después de conocer el libro de Giustini, diez años, que sin duda fueron los de su madurez humana y artística. Pero no puede dudarse de que para 1732 tuviera compuestas ya muchas de sus sonatas. Con todo, algunas las habrá compuesto, sin duda, después de esa fecha. De hecho, en algunas parece vislumbrarse un cambio en su concepción de la sonata. Por ejemplo, en la $n .^{\circ} 25$, en re menor, se encuentran tantos elementos nuevos, que por fuerza tuvo que haber algo antes de ella, para que Seixas concibiera esa obra. Y no estaría de más recordar que se trata de una sonata en tres tiempos; que el primero no tiene señal de tempo - el "allegro" que Kastner le anadió me parece algo rápido en demasía; y creo que un "andante con motto", o algo así, reflejaría mejor, creo yo, el espíritu tan notable de ese movimiento. El segundo tiempo es un adagio. El tercero el habitual minueto. Véase el comienzo del primer movimiento:

(15) Lo más notable es que esto mismo hicieron los compositores españoles de la época de Carlos Seixas o ligeramente posteriores que también escribieron sonatas para "címbalo" y para "piano-forte", sobre todo Albero y Rodríguez Monllor. El estudio comparativo entre su concepción de la sonata, junto con el lenguaje que utilizan, y lo realizado por Seixas es sumamente ilustrativo y demuestra que había en el ambiente ibérico algo que hacía que todos nuestros compositores reaccionaran de una manera esencialmente idéntica a los nuevos conceptos musicales que entonces estaban naciendo, sobre todo en Italia. 

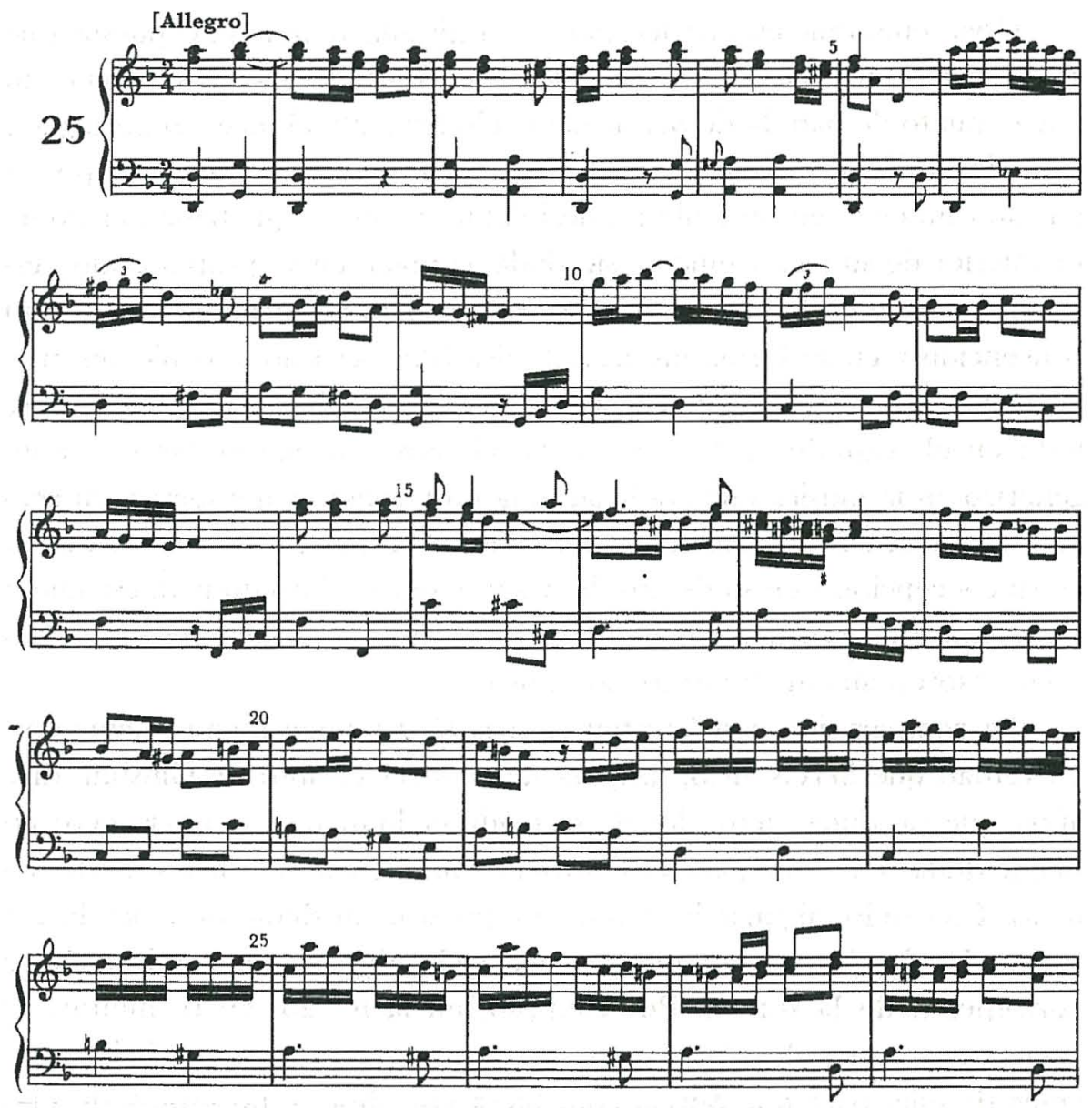

Ej. 7. Carlos Seixas: Sonata 25, en re menor. Primer movimiento.

De nuevo hay que decir que sí, que se percibe en algunas sonatas de Seixas un cierto influjo de las de Giustini; pero es igualmente claro que ese influjo queda del todo eclipsado por la personalidad artística del gran compositor portugués.

Lo que sí hay que decir, como conclusión, es que estamos en un mundo del todo diverso del de Roque da Conceição y sus contemporáneos. Estamos, para decirlo con la expresión que usé al comienzo de esta exposición, en la música "moderna", frente a la "antigua" de finales del siglo XVII.

Queda una segunda parte, no menos instructiva que esta primera que hemos tratado, para completar el conocimiento de lo que significó Seixas 
en la Península Ibérica, y aun para desarrollar adecuadamente el título de la presente comunicación: enmarcar la obra teclística de Seixas en la ibérica de la segunda mitad del siglo, tanto de Portugal - Francisco Xavier Baptista, João Domingos Bontempo, João Sousa Carvalho, Manuel de Santo Elías... - como en la de España - Sebastián Albero, Vicente Rodríguez Monllor, los Nebra... -; pero eso no es posible aquí, ya que exigiría un espacio similar al ya utilizado, por lo que deberá ser dejado para otra ocasión, que espero sea próxima.

No me queda sino agradecer, una vez más, a la Universidad de Coimbra y al Dr. Pedrosa el haberme invitado a este acto, y a todos ustedes por su atención.

Muchas gracias a todos. 


\section{Série}

\section{Documentos}

Imprensa da Universidade de Coimbra

Coimbra University Press

2004

- U

C • 